\title{
Zeeman Splitting of Zero-Dimensional Heavy-Hole States in a Strongly Strained Ge Quantum Well
}

O. B. Agafonov, K.-M. Haendel, R. Winkler, U. Denker, O. G. Schmidt, and R. J. Haug

Citation: AIP Conference Proceedings 893, 1369 (2007); doi: 10.1063/1.2730413

View online: https://doi.org/10.1063/1.2730413

View Table of Contents: http://aip.scitation.org/toc/apc/893/1

Published by the American Institute of Physics

\section{Articles you may be interested in}

SiGe quantum dots for fast hole spin Rabi oscillations

Applied Physics Letters 103, 263113 (2013); 10.1063/1.4858959

Pauli blockade in a few-hole PMOS double quantum dot limited by spin-orbit interaction

Applied Physics Letters 109, 193101 (2016); 10.1063/1.4966946

Measuring hole spin states of single quantum dot in germanium hut wire

Applied Physics Letters 110, 133105 (2017); 10.1063/1.4979521

\section{Conference Proceedings}

\section{Get $30 \%$ off all print proceedings!}

Enter Promotion Code PDF-30 at checkout 


\title{
Zeeman Splitting of Zero-Dimensional Heavy-Hole States in a Strongly Strained Ge Quantum Well
}

\author{
O. B. Agafonov*, K.-M. Haendel*, R. Winkler ${ }^{\Uparrow}$, U. Denker**, O. G. Schmidt** \\ and R. J. Haug* \\ *Institut für Festkörperphysik, Leibniz Universität Hannover, Appelstraße 2, D-30167 Hannover \\ 'Department of Physics, Northern Illinois University, De Kalb, IL 60115 \\ **Max-Plank-Institut für Festkörperforschung, Heisenbergstraße 1, D-70569 Stuttgart
}

\begin{abstract}
The method of magnetotunneling spectroscopy has been used for experimental probing of heavy-hole impurity states in $\mathrm{Si} / \mathrm{Ge}$ double-barrier heterostructures in magnetic field up to 18 Tesla. The impurities were located in a strained Ge quantum well with a thickness of four monolayers. We have observed a giant anisotropy of Zeeman splitting for these zero-dimensional systems. The splitting was measured as a function of angle between the external magnetic field and the quantum well plane. A complete suppression of the splitting takes place when the magnetic field is oriented parallel to the sample surface and quantum well plane, while in the perpendicular field the observed splitting is maximal.
\end{abstract}

Keywords: Quantum well, magnetic field, spin-orbit effects.

PACS: R71.18.+y, 71.70.Fk, 73.21.Fg

\section{INTRODUCTION}

Spin-related phenomena in semiconductors draw attention of many researchers due to their possible application in spintronic devices and for quantum computing. A lot of these investigations are devoted to the study of spin-orbit interaction and to spin manipulation along with effective Landé factor $g$ engineering. We report the results of our study of the influence of homogeneous magnetic fields $B$ up to 18 Tesla on the current-voltage characteristics (IVC) of a resonant tunneling $\mathrm{Si} / \mathrm{Ge}$ heterostructure in the temperature range from $100 \mathrm{mK}$ to $1 \mathrm{~K}$.

\section{EXPERIMENT}

The measurements were carried out on the samples fabricated of a $\mathrm{Si} / \mathrm{Ge}$ double-barrier heterostructure with a 4 monolayer-thick strained Ge quantum well embedded in $\mathrm{Si}$. This structure was grown by molecular-beam epitaxy on a boron-doped $p^{+}-\mathrm{Si}$ (100) substrate at $460^{\circ} \mathrm{C}$. A series of vertical resonant tunneling diodes with the average in-plane dimensions of order of $1 \mu \mathrm{m}$ were processed using electron-beam lithography. The schematic picture of the layer sequence of the structure used is given in Fig. 1 along with the theoretically calculated valence band profile.

The orientation of the external magnetic field $B$ applied to the samples was varied in the range from $\Theta$ $=0^{\circ}$ to $\Theta=90^{\circ}$, where $\Theta$ is the angle between the field direction and the quantum well plane.

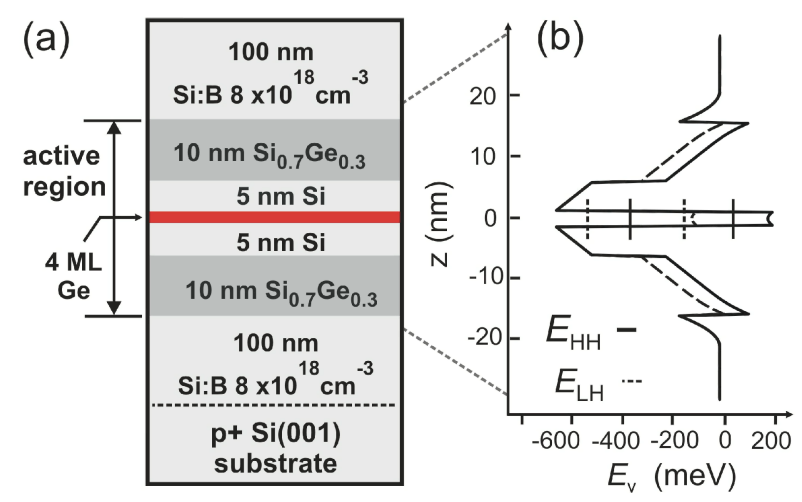

FIGURE 1. (a) Layer sequence of the studied $\mathrm{Si} / \mathrm{Ge}$ heterostructure. (b) Self-consistently calculated valence band profile of an active region. The solid line illustrates the course of the heavy-hole subband $\mathrm{E}_{\mathrm{HH}}$. The dashed line corresponds to the light-hole subband $\mathrm{E}_{\mathrm{LH}}$. 


\section{RESULTS AND DISCUSSION}

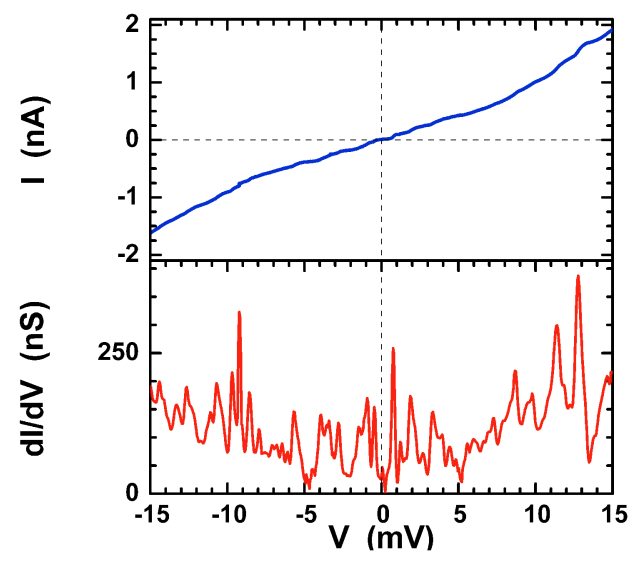

FIGURE 2. (a) Typical current-voltage characteristic of resonant tunneling diode at temperature $100 \mathrm{mK}$ and $\mathrm{B}=0 \mathrm{~T}$. (b) Differential conductance $\mathrm{dI} / \mathrm{dV}$.

The current-voltage characteristics of the samples reveal at low temperatures step-like features [1]. This behavior is observable at bias voltages much lower than voltages (of order of $300 \mathrm{mV}$ ) at which an onset of resonant tunneling of holes through two-dimensional states of the Ge quantum well sets in. We attribute these current steps to the tunneling process of holes through the zero-dimensional (0D) energy states created by boron dopant-atoms which have migrated from highly-doped contact layers into the region of the Ge quantum well. Tunneling through $0 \mathrm{D}$ heavy-hole $(\mathrm{HH})$ state $E_{\mathrm{HH}}$ in the quantum well takes place each time $E_{\mathrm{HH}}$ is in resonance with the Fermi energy of emitter. An example of typical IVC inherent to our diodes is shown in Fig. 2 along with the corresponding differential conductance $d I / d V$.

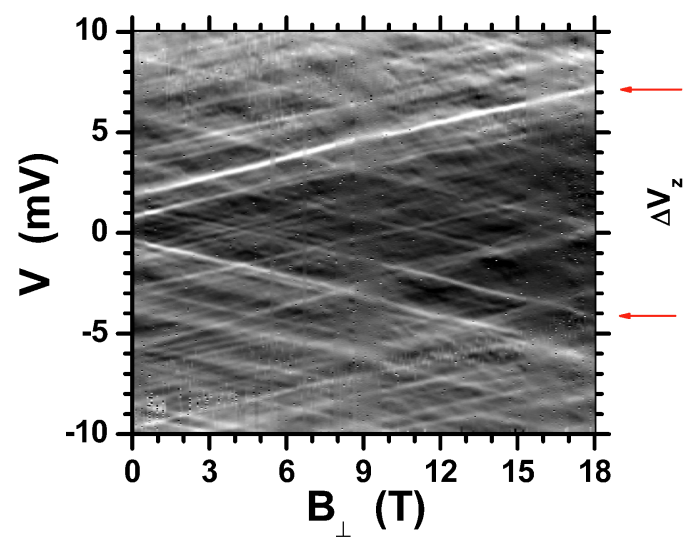

FIGURE 3. Gray-scale plot of differential conductance $\mathrm{dI} / \mathrm{dV}$ at the temperature $100 \mathrm{mK}$ measured in the magnetic field orientated perpendicular to the quantum well plane.

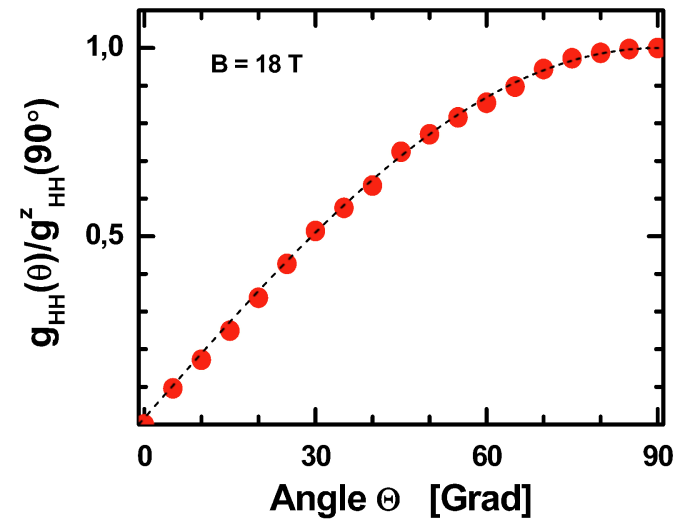

FIGURE 4. Angular dependence of $g$-factor of heavy-holes in the tilted magnetic field at $18 \mathrm{~T}$. $\Theta$ is the angle between magnetic field and quantum well plane.

From the temperature-dependent broadening of the current step edges we have determined the energy-to-bias conversion factor [2] $\alpha \cong 0.5$.

In the magnetic field directed perpendicular to the quantum well plane we observed a linear splitting of differential conductance $d I / d V$ peaks (see Fig. 3) attributed to the Zeeman splitting of heavy-hole states in Ge quantum well.

$$
\Delta E_{z}=g_{H H}^{z} \mu_{B} B_{\perp} .
$$

As we see all levels reveal the same splitting. The gradient of the splitting $d \Delta V_{\mathrm{z}} / d B_{\perp}=0.73 \mathrm{mV} / \mathrm{T}$. The value of $g$-factor for this field orientation was determined as

$$
g_{H H}^{z}=\left(\alpha e / \mu_{B}\right) \cdot\left(d \Delta V_{z} / d B_{\perp}\right)=6.3 \text {. }
$$

The angular dependence of $g_{\mathrm{HH}}$ in the tilted magnetic field at $18 \mathrm{~T}$ is plotted in Fig. 4. The splitting turned out to be strongly anisotropic. In the perpendicular field $B_{\perp}$ the splitting is maximal. In an in-plane field $B_{\|}$we observed a complete suppression of the splitting. This result correlates with the in [3] reported evidence of $\mathrm{HH}$ spin splitting which is determined only by $B_{\perp}$ component of magnetic field.

\section{REFERENCES}

1. K. -M. Haendel, R. Winkler, U. Denker, O. G. Schmidt, and R. J. Haug, Phys. Rev. Lett. 96, 086403 (2006).

2. B. Su, V. J. Goldman, and J. E. Cunningham, Phys. Rev. B 46, 7644 (1992).

3. R. W. Martin, R. J. Nicholas, G. J. Rees, N. J. Mason, and P. J. Walker, Phys. Rev. B 42, 9237 (1990). 